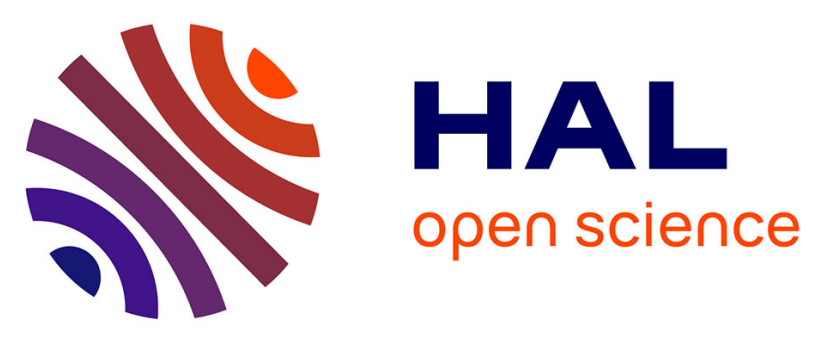

\title{
Cellulitis of the face associated with SENLAT caused by Rickettsia slovaca detected by qPCR on scalp eschar swab sample: An unusual case report and review of literature
}

Marie Hocquart, Hortense Drouet, Paul Levet, Didier Raoult, Philippe Parola, Carole Eldin

\section{To cite this version:}

Marie Hocquart, Hortense Drouet, Paul Levet, Didier Raoult, Philippe Parola, et al.. Cellulitis of the face associated with SENLAT caused by Rickettsia slovaca detected by qPCR on scalp eschar swab sample: An unusual case report and review of literature. Ticks and Tick-borne Diseases, 2019, 10 (5), pp.1142-1145. 10.1016/j.ttbdis.2019.06.010 . hal-02465936

\section{HAL Id: hal-02465936 \\ https://hal-amu.archives-ouvertes.fr/hal-02465936}

Submitted on 25 Oct 2021

HAL is a multi-disciplinary open access archive for the deposit and dissemination of scientific research documents, whether they are published or not. The documents may come from teaching and research institutions in France or abroad, or from public or private research centers.
L'archive ouverte pluridisciplinaire HAL, est destinée au dépôt et à la diffusion de documents scientifiques de niveau recherche, publiés ou non, émanant des établissements d'enseignement et de recherche français ou étrangers, des laboratoires publics ou privés.

\section{(ㅇ)(1) $\$$}

Distributed under a Creative Commons Attribution - NonCommerciall 4.0 International 
1 Cellulitis of the face associated with SENLAT caused by Rickettsia slovaca detected by

2 qPCR on scalp eschar swab sample: an unusual case report and review of literature

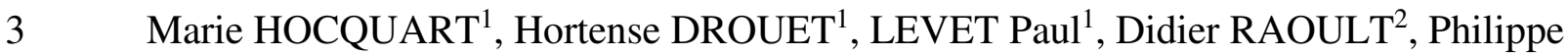

4 PAROLA $^{1}$, Carole ELDIN $^{1}$

$5 \quad{ }^{1}$ Aix Marseille Univ, IRD, AP-HM, SSA, VITROME, IHU-Méditerranée Infection,

6 Marseille, France.

$7 \quad{ }^{2}$ Aix Marseille Univ, IRD, AP-HM, MEPHI, IHU-Méditerranée Infection,

8 Marseille, France.

9 Corresponding author e-mail: carole.eldin@gmail.com

10 Full postal adress: Institut Hospitalo-Universitaire Méditerranée Infection, 19-21 boulevard 11 Jean Moulin, 13005 Marseille, France

12 Phone: [33] 413732001

13 Abstract word count: 183

14 Body text word count: 1995

15 Keywords: R. slovaca, SENLAT syndrome, Periorbital Cellulitis

16 


\section{Abstract}

18 Background: Tick-borne rickettsioses are infectious diseases caused by obligate intracellular

19 Gram-negative bacteria belonging to the spotted fever group of Rickettsia.

20 Methods: We describe an unusual case of SENLAT (Scalp eschar and neck lymphadenopathy

21 after tick bite), caused by Rickettsia slovaca, associated with a cellulitis of the face in a 70-

22 year-old woman, and diagnosed using qPCR on a scalp eschar swab. We review the literature

23 regarding cases of SENLAT-associated-cellulitis and case of SENLAT diagnosed by qPCR

24 on scalp eschar swabs.

25 Results: We found only one previous report of SENLAT associated with a cellulitis of the

26 face. It was a nine-year-old French girl diagnosed by seroconversion for Rickettsia sp. Our

27 review of the literature showed that qPCR on eschar swab samples is a less invasive method

28 than performing cutaneous biopsy of the eschar and has good sensitivity and specificity (90\%

29 and $100 \%$ respectively).

30 Conclusions: We report the second case of cellulitis of the face associated with the SENLAT

31 syndrome. Detection of Rickettsia by qPCR on swab sample of the scalp eschar is a simple,

32 noninvasive technique allowing rapid diagnosis and treatment when SENLAT is suspected. 
Tick-borne rickettsioses are infectious diseases caused by obligate intracellular Gramnegative bacteria of the spotted fever group (SFG), belonging to the genus Rickettsia in the order Rickettsiales. Hard ticks may act as vectors, reservoirs, and/or amplifiers of SFG rickettsiae (Delord et al., 2014). The pathogenic role of Rickettsia slovaca was first demonstrated in 1997 in a patient who presented with a single inoculation lesion of the scalp and enlarged cervical lymph nodes after being bitten by a Dermacentor tick (Raoult et al., 2002). The acronym TIBOLA (tick-borne lymphadenopathy) was proposed because of painful lymphadenopathy next to the region of the tick bite. Later, a similar clinical entity was described and named DEBONEL (Dermacentor-borne necrosis erythema lymphadenopathy) because, although $R$. slovaca is frequently involved, there have been reports describing other bacteria, such as $R$. raoultii, $R$. sibirica mongolitimonae, $R$. massiliae, Bartonella henselae, Francisella tularensis. (Angelakis et al., 2010; Dubourg et al., 2014). “Candidatus Rickettsia rioja", Coxiella burnetii and Borrelia burgdorferi sensu lato are possible etiological agents due to indirect molecular evidence in ticks involved in SENLAT cases (Dubourg et al., 2014). However, in some cases (20-25\%), the causative agent remains undetermined suggesting that other microorganisms might be involved (Dubourg, G. et al. 2014). Rickettsia slovaca, the most frequent reported agent of SENLAT, is transmitted by ticks of the genus Dermacentor and especially by D. marginatus (Supplementary Figure 1) or $D$. reticulatus. The geographical distribution of $R$. slovaca most likely corresponds to the geographical distribution of these ticks in Europe and North Africa (Dubourg et al., 2014). Rickettsia slovaca has also been found in D. marginatus ticks in the Kurgan region (Ural) of

60 Russia and in Georgia, and in $6.5 \%$ of D. silvarum ticks in China. Human cases have not been 
reported in Asia (Parola et al., 2013). Infection is most frequent among women (67 to 100\%) and children under 12 years old (41 to $43 \%$ ). The median incubation period is of 5 to 10 days (range, 1 to 15 days). In Europe, infections occur most frequently from March to May and from September to November, which corresponds to the periods of greatest activity of Dermacentor adult ticks (Parola et al., 2013). Rickettsia slovaca has also been reported as an agent of fever of unknown origin (Botelho-Nevers and Raoult, 2007) among travelers returning from Mongolia, Italy and Corsica (Delord et al., 2014).

Clinical manifestations of SENLAT include inoculation lesion at the upper half of the body, mainly on scalp in humans, probably because Dermacentor ticks are used to parasitize long-haired animals like horses and wild boar(Parola et al., 2009). The inoculation lesion is often centered by the tick bite with an erythema of at least $5 \mathrm{~mm}$ that encircles the lesion and second cervical or occipital lymphadenopathies, satellite of the eschar, which may be large, inflammatory and painful (Parola et al., 2009). These two elements may be associated with fever, rash, localized alopecia, headache, asthenia, sweats, arthralgia and myalgia (Dubourg et al., 2013).

$$
\text { We describe an unusual case of SENLAT associated with a cellulitis of the face }
$$
diagnosed by qPCR on a scalp eschar swab and review the literature about this entity and the diagnosis by qPCR on eschar swabs.

(1)

\section{The Case}

A 70-year-old woman with medical history of hypothyroidism was admitted in our department in Marseille on April 2017 with a periorbital cellulitis associated with a scalp eschar and cervical lymphadenopathies. The patient reported a walk in the forest about ten days ago with the apparition, a few days later, of odynophagia, bilateral cervical lymphadenopathies and lesion of the scalp, without fever. Her family doctor thought she had 
tonsillitis and prescribed amoxicillin. Subsequently, a large erythema and edema of the face appeared, so that she consulted to our unit. At physical examination we recorded a scalp eschar on the left side of the head, an erythema and edema at the forehead with rapid extension to periorbital, retro auricular and cervical areas with left-sided lymphadenopathies (Figure 1). Laboratory tests were normal except a slight increase in the C-reactive- protein (15mg/l). Cervical and face tomodensitometry showed a cellulitis without fascia involvement or abscess. Empirical antibiotherapy with amoxicillin- clavulanic acid and clindamycin had been started before at the emergency unit and we added oral doxycycline (200mg/daily) for ten days because of the presence of the inoculation eschar on the scalp. A dry sterile swab sample was collected from the inoculation eschar. The swab, while being rotated vigorously, was directed to the base of the eschar at a $50^{\circ}-60^{\circ}$ angle for 5-6 times (Mouffok et al., 2011). qPCR on scalp eschar swab targeting a fragment of the gltA gene, as previously described (Aubry et al., 2016), was positive for Rickettsia sp. and sequencing of a fragment of the ompA gene (Aubry et al., 2016) identified Rickettsia slovaca. Culture of the scalp swab sample on human embryonic lung cells using shell vial methods as previously reported (Raoult et al., 2002) were negative. Serum sample were analysed using indirect Immunofluorescence Assay (IFA) against two spotted fever group rickettsial antigens (R.conorii conorii, Rickettsia felis) and a typhus group antigen (R.typhi). The IFA test during the acute phase was considered positive if antibody titres were $>$ 1:128 for immunoglobulin $G(\operatorname{IgG})$ and $>1: 64$ for immunoglobulin $\mathrm{M}(\operatorname{IgM})$ for the spotted fever group, and > 1:64 for IgG and > 1:32 for typhus group, as previously described (Hayet et al., 2018). These serological testings were negative in the acute phase serum sample and in a convalescent serum sample one month after the resolution of symptoms. The patient completely recovered after ten days of doxycycline except for a small cicatricial alopecia at the site of the eschar (Figure 1). 
In 2009, Parola et al. described facial edema without cellulitis as a new clinical feature

112 of SENLAT because it was found in $19 \%$ of cases of $R$. slovaca and $40 \%$ of $R$. raoultii

113 infections (Parola et al., 2009). A literature search revealed only one previous reference

114 reporting a case of SENLAT associated with a similar cellulitis of the face. It was a nine-year-

115 old French girl with an acute hemifacial edema and erythema requalified as TIBOLA in 2011

116 (Gaston et al., 2011). The removal of two ticks in the scalp had occurred 10 days earlier. The

117 edema appeared suddenly in a context of fever at $39.5^{\circ} \mathrm{C}$, headache and vertigo. The clinical

118 examination revealed an erysipeloid placard of the left hemiface, associated with a

119 homolateral jugulo-carotid inflammatory adenitis. At the same time, the examination of the

120 scalp revealed two necrotic eschars surrounded by an erythematous halo. The diagnosis was

121 done by seroconversion for Rickettsia fifteen days later (Gaston et al., 2011).

122 The microbiological methods available for the diagnosis of tick-borne rickettsioses are

123 serology, culture and PCR. Serology is the most widespread method for the diagnosis of tick-

124 borne rickettsioses, but seroconversion occurs only 15 to 21 days after the beginning of the

125 symptoms (Foissac et al., 2013). Moreover, even when using IFA, which is the reference

126 method, cross reactions are frequent and the specific serological sensitivity for $R$. slovaca is

127 low (about 12\%), probably due to a limited locoregional dissemination of the infection

128 (Foissac et al., 2013), as illustrated by our case, where even convalescent serum sample was

129 negative. Culture is fastidious and requires cell cultures that are only performed in specialized

130 BSL 3 laboratories. On the other hand, molecular techniques (qPCR targeting the gltA gene

131 for Rickettsia group and species-specific qPCR) are useful given their good sensitivity at the

132 beginning of the infection, especially on eschar samples (Foissac et al., 2013). qPCR on a

133 swab sample of the eschar requires the use of a sterile swab to obtain a sample from inside the

134 eschar, simply by inserting the swab into the eschar and rotating it to the basis of the eschar.

135 The material obtained via swabbing can be then processed for nucleic acid extraction and 
subsequent PCR analysis (Luce-Fedrow et al., 2015). It has a sensitivity of 85 to $90 \%$ and a specificity close of 100\% (Bechah et al., 2011; Mouffok et al., 2011; Renvoise et al., 2012; Wang et al., 2009). These performances are almost comparable to the ones of qPCR on eschar cutaneous biopsy (sensitivity $>95 \%$ and specificity $>98 \%$ ) (Foissac et al., 2013). qPCR on eschar swab samples has been used with success around the world for detection of $R$. parkeri, R. conorii, $R$. sibirica mongolitimonae, $R$. africae, $R$. australis, $R$. felis and $O$. tsutsugamushi

(Table 1). To date, only 2 references reported the detection of $R$. slovaca by qPCR on a swab eschar sample (Bechah et al., 2011; Dubourg et al., 2014). Swabbing an eschar has a lot of advantages because it is a noninvasive, simple, rapid and painless technique that can be easily performed without risk, especially in children. This test can be used at the bedside or in an outpatient clinic and could be useful for epidemiologic, surveillance and clinical studies even although health care workers are not able to perform biopsies (Le Viet et al., 2017; LuceFedrow et al., 2015; Renvoise et al., 2012). In a recent study, opinions of health care providers and patients were evaluated and they both preferred collecting swab samples over biopsy samples (46 vs. 5 and 57 vs. 2, respectively; $p=0.0001$ ) (Mouffok et al., 2011). In a clinical setting, the treatment of choice for SFG rickettsia is doxycycline (Botelho-Nevers et al., 2012), including in children for whom its safety has been demonstrated, notably on the absence of dental damage (Todd et al., 2015; Volovitz et al., 2007). There is no data in the literature to determine the exact duration of treatment, which is related to the clinical response and is classically of 7 to 14 days (Foissac et al., 2013), or at least 3 days after apyrexia (Renvoise et al., 2009). The complete resolution of symptoms is long but antibiotic treatment can reduce the duration of symptoms from 62 to 50 days (Foissac et al., 2013). Sequelar alopecia at the tick bite site, as observed in our patient, is reported in 20 to $51 \%$ of cases (Foissac et al., 2013). 
161 cannot be used to evaluate the efficacy of antibiotics. Cell culture techniques are used to test

162 in vitro susceptibility of Rickettsia to antibiotics by determining the minimum inhibitory

163 concentrations but can be performed only in specialized laboratories (Renvoise et al., 2009).

164 However, beta-lactams, aminoglycosides and cotrimoxazole are ineffective on rickettsia

165 (Renvoise et al., 2009). As a consequence, in a patient with a cellulitis, the presence of an

166 eschar and lymphadenopathies should prompt clinicians to add doxycycline, because beta

167 lactam are inefficient on SENLAT-associated cellulitis.

\section{Conflict of interest}

169 All the authors have no conflict of interest to disclose

170 Funding source

171 This research did not receive any specific grant from funding agencies in the public,

172 commercial, or not-for-profit sectors.

\section{Aknowledgements}

174 None 
Angelakis, E., Pulcini, C., Waton, J., Imbert, P., Socolovschi, C., Edouard, S., Dellamonica,

179 P., \& Raoult, D. 2010. Scalp eschar and neck lymphadenopathy caused by Bartonella

180 henselae after Tick Bite. Clin.Infect.Dis., 50(4): 549-551.

181 Aubry, C., Socolovschi, C., Raoult, D., \& Parola, P. 2016. Bacterial agents in 248 ticks

182 removed from people from 2002 to 2013. Ticks.Tick.Borne.Dis., 7(3): 475-481.

183 Bechah, Y., Socolovschi, C., \& Raoult, D. 2011. Identification of rickettsial infections by

184 using cutaneous swab specimens and PCR. Emerg.Infect.Dis., 17(1): 83-86.

185 Botelho-Nevers, E. \& Raoult, D. 2007. Fever of unknown origin due to rickettsioses.

186 Infect.Dis.Clin.North Am., 21(4): 997-1011, ix.

187 Botelho-Nevers, E., Socolovschi, C., Raoult, D., \& Parola, P. 2012. Treatment of Rickettsia

188 spp. infections: a review. Expert.Rev.Anti.Infect.Ther., 10(12): 1425-1437.

189 Delord, M., Socolovschi, C., \& Parola, P. 2014. Rickettsioses and Q fever in travelers (2004190 2013). Travel.Med.Infect.Dis., 12(5): 443-458.

191 Dubourg, G., Socolovschi, C., Del, G. P., Fournier, P. E., \& Raoult, D. 2014. Scalp eschar

192 and neck lymphadenopathy after tick bite: an emerging syndrome with multiple causes. Eur.J

193 Clin.Microbiol.Infect.Dis., 33(8): 1449-1456.

194 Foissac, M., Socolovschi, C., \& Raoult, D. 2013. [Update on SENLAT syndrome: scalp

195 eschar and neck lymph adenopathy after a tick bite]. Ann.Dermatol.Venereol., 140(10): 598-

196609.

197 Gaston, J., Durox, H., Sparsa, A., Bonnetblanc, J. M., \& Doffoel-Hantz, V. 2011.

198 [Dermohypodermitis on the face revealing TIBOLA]. Arch.Pediatr., 18(5): 565-567.

199 Bouchaib, H., Eldin, C., Laroche, M., Raoult, D., \& Parola, P. 2018. Tick-and flea-borne 200 rickettsioses in Tizi-Ouzou, Algeria: Implications for travel medicine. Travel medicine and 201 infectious disease, 26, 51-57.

202 Khrouf, F., Sellami, H., Elleuch, E., Hattab, Z., Ammari, L., Khalfaoui, M., Souissi, J., 203 Harrabi, H., M'ghirbi, Y., Tiouiri, H., Ben, J. M., Hammami, A., Letaief, A., Bouattour, A., \& 204 Znazen, A. 2016. Molecular diagnosis of Rickettsia infection in patients from Tunisia.

205 Ticks.Tick.Borne.Dis., 7(5): 653-656.

206 Kim, D. M., Kim, H. L., Park, C. Y., Yang, T. Y., Lee, J. H., Yang, J. T., Shim, S. K., \& Lee, 207 S. H. 2006. Clinical usefulness of eschar polymerase chain reaction for the diagnosis of scrub 208 typhus: a prospective study. Clin.Infect.Dis., 43(10): 1296-1300.

209 Le, V. N., Laroche, M., Thi Pham, H. L., Viet, N. L., Mediannikov, O., Raoult, D., \& Parola, 210 P. 2017. Use of eschar swabbing for the molecular diagnosis and genotyping of Orientia 211 tsutsugamushi causing scrub typhus in Quang Nam province, Vietnam. PLoS.Negl.Trop.Dis., 212 11(2): e0005397. 
Luce-Fedrow, A., Mullins, K., Kostik, A. P., St John, H. K., Jiang, J., \& Richards, A. L. 2015. Strategies for detecting rickettsiae and diagnosing rickettsial diseases. Future.Microbiol., 10(4): 537-564.

Mediannikov, O., Socolovschi, C., Million, M., Sokhna, C., Bassene, H., Diatta, G., Fenollar, F., \& Raoult, D. 2014. Molecular identification of pathogenic bacteria in eschars from acute febrile patients, Senegal. Am.J Trop.Med.Hyg., 91(5): 1015-1019. rickettsioses from eschar swab samples, Algeria. Emerg.Infect.Dis., 17(10): 1968-1969.

Myers, T., Lalani, T., Dent, M., Jiang, J., Daly, P. L., Maguire, J. D., \& Richards, A. L. 2013. Detecting Rickettsia parkeri infection from eschar swab specimens. Emerg.Infect.Dis., 19(5): 778-780.

Parola, P., Paddock, C. D., Socolovschi, C., Labruna, M. B., Mediannikov, O., Kernif, T., Abdad, M. Y., Stenos, J., Bitam, I., Fournier, P. E., \& Raoult, D. 2013. Update on tick-borne rickettsioses around the world: a geographic approach. Clin.Microbiol.Rev., 26(4): 657-702.

Parola, P., Rovery, C., Rolain, J. M., Brouqui, P., Davoust, B., \& Raoult, D. 2009. Rickettsia slovaca and R. raoultii in tick-borne Rickettsioses. Emerg.Infect.Dis., 15(7): 1105-1108.

Raoult, D., Lakos, A., Fenollar, F., Beytout, J., Brouqui, P., \& Fournier, P. E. 2002. Spotless rickettsiosis caused by Rickettsia slovaca and associated with Dermacentor ticks.

Renvoise, A. \& Raoult, D. 2009. [An update on rickettsiosis]. Med.Mal Infect., 39(2): 71-81.

Renvoise, A., Rolain, J. M., Socolovschi, C., \& Raoult, D. 2012. Widespread use of real-time PCR for rickettsial diagnosis. FEMS Immunol.Med.Microbiol., 64(1): 126-129.

Socolovschi, C., Renvoise, A., Brouqui, P., Parola, P., \& Raoult, D. 2012. The use of eschar swabs for the diagnosis of African tick-bite fever. Ticks.Tick.Borne.Dis., 3(5-6): 361-363.

Solary, J., Socolovschi, C., Aubry, C., Brouqui, P., Raoult, D., \& Parola, P. 2014. Detection of Rickettsia sibirica mongolitimonae by using cutaneous swab samples and quantitative PCR. Emerg.Infect.Dis., 20(4): 716-718.

Todd, S. R., Dahlgren, F. S., Traeger, M. S., Beltran-Aguilar, E. D., Marianos, D. W., Hamilton, C., McQuiston, J. H., \& Regan, J. J. 2015. No visible dental staining in children treated with doxycycline for suspected Rocky Mountain Spotted Fever. J Pediatr., 166(5): $1246-1251$.

Volovitz, B., Shkap, R., Amir, J., Calderon, S., Varsano, I., \& Nussinovitch, M. 2007. Absence of tooth staining with doxycycline treatment in young children. Clin.Pediatr.(Phila), 46(2): 121-126.

Wang, J. M., Hudson, B. J., Watts, M. R., Karagiannis, T., Fisher, N. J., Anderson, C., \& Roffey, P. 2009. Diagnosis of Queensland tick typhus and African tick bite fever by PCR of 
Table 1: Agents of Rickettsia spp. detected by qPCR on eschar swab

\begin{tabular}{|c|c|c|c|}
\hline Agents & Authors & $\begin{array}{l}\text { Positive } \\
\text { SWAB }\end{array}$ & Clinical presentation/ Localization of eschar \\
\hline R. parkeri & Myers et al., 2013 & 2 & $\begin{array}{l}\text { Fever, chills, night sweats, a diffuse maculopapular rash, headache, myalgia, neck stiffness, } \\
\text { arthralgia, and malaise. }\end{array}$ \\
\hline \multirow{3}{*}{ R. conorii } & Bechah et al., 2011 & 25 & Fever, eschar and generalized maculopapular rash. \\
\hline & Khrouf et al., 2016 & 12 & Acute fever and cutaneous rashes and/or eschars. \\
\hline & Mouffok et al., 2011 & 2 & NS \\
\hline \multirow[t]{2}{*}{ R. sibirica mongolitimonae } & Bechah et al., 2011 & 2 & NS \\
\hline & Solary et al., 2014 & 1 & Fever, 2 eschars on the lower right eyelid, periorbital edema, cervical lymphadenopathies. \\
\hline \multirow[t]{2}{*}{ R. africae } & Bechah et al., 2011 & 2 & NS \\
\hline & Socolovschi et al., 2012 & 4 & Fever, eschar, rash, lymph-adenopathy \\
\hline R. australis & Bechah et al., 2011 & 1 & NS \\
\hline
\end{tabular}


Wang et al., 2009

R. felis

O. tsutsugamushi

Kim et al., 2006

Le Viet et al., 2017

R. slovaca

Bechah et al., 2011

Dubourg et al., 2014
Case 1: inguinal eschar, tender local lymphadenopathy, fever to $39.8^{\circ} \mathrm{C}$, severe headache, myalgia, arthralgia, and generalized sparse rash

Case 2: Fever, torso eschar, severe headache, myalgia, arthralgia, and rash Case 3: Fever, eschar on the scalp, tender local lymphadenopathy, and generalized rash with maculopapular and vesicular components.

Case 4: Fever, eschar on the torso, tender local lymphadenopathy, and generalized sparse rash characterized by papular and vesiculopustular lesions.

Fever and eschar.

Fever with eschar or a maculopapular skin rash and also had > 2 symptoms (such as headache, malaise, myalgia, coughing, nausea, and abdominal discomfort)

$>15$ years, axillary temperature $>37.5^{\circ} \mathrm{C}$ and at least one of the following four secondary findings: eschar, skin rash, lymphadenopathy, hepatomegaly and/or splenomegaly

4 Fever, inoculation lesion, enlarged nodes, localized alopecia

$1 \quad$ NS 


\section{Figures legends}

\section{Figure 1}

Scalp eschar (Panel a) and peri-orbital cellulitis of the face before (Panel b) and after treatment (Panel c) in a 70-year-old women, revealing a SENLAT syndrome caused by $R$. slovaca detected by qPCR on scalp eschar swab. 


\section{Supplementary Figure}

\section{Supplementary Figure 1}

Dermacentor marginatus ticks: female (Panel a) and male (Panel b). 

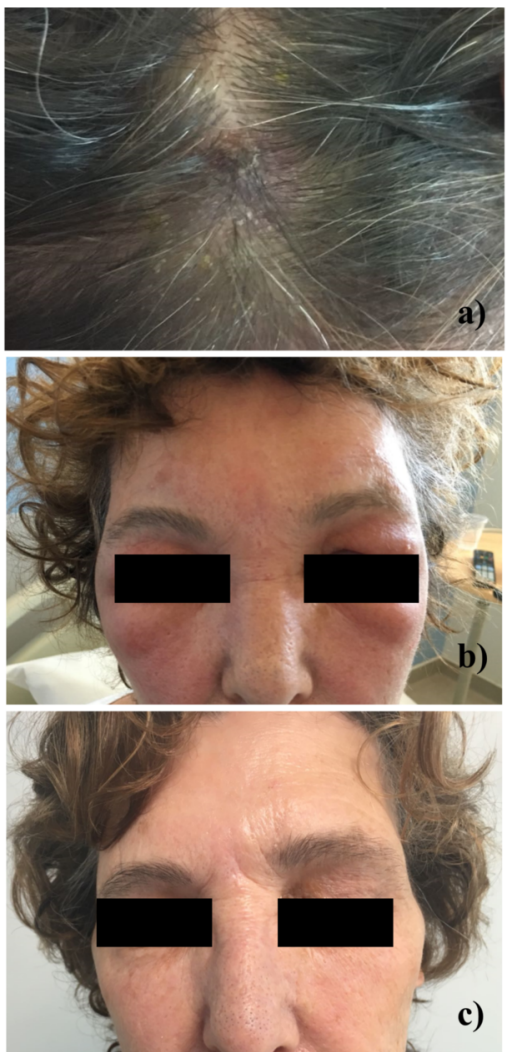